\title{
BMJ Open Oral health interventions for older people in residential aged care facilities: a protocol for a realist systematic review
}

\author{
Amanda Kenny, ${ }^{1}$ Virginia Dickson-Swift (10 , ${ }^{1}$ Carina Ka Yee Chan, ${ }^{2}$ Mohd Masood, ${ }^{3}$ \\ Mark Gussy, ${ }^{4}$ Bradley Christian, ${ }^{3}$ Brad Hodge, ${ }^{1}$ Susan Furness, ${ }^{1}$ \\ Lisa C Hanson (10) , ${ }^{1}$ Samantha Clune, ${ }^{1}$ Emma Zadow, ${ }^{1}$ Ron J Knevel ${ }^{3}$
}

To cite: Kenny A, DicksonSwift V, Chan CKY, et al. Oral health interventions for older people in residential aged care facilities: a protocol for a realist systematic review. BMJ Open 2021;11:e042937. doi:10.1136/ bmjopen-2020-042937

- Prepublication history for this paper is available online. To view these files, please visit the journal online (http://dx.doi org/10.1136/bmjopen-2020042937).

Received 20 July 2020 Revised 08 April 2021 Accepted 16 April 2021

\section{Check for updates}

(c) Author(s) (or their employer(s)) 2021. Re-use permitted under CC BY-NC. No commercial re-use. See rights and permissions. Published by BMJ.

${ }^{1}$ La Trobe Rural Health School, La Trobe University, Bendigo, Victoria, Australia

${ }^{2}$ School of Psychology and Public Health, La Trobe University College of Science, Health and Engineering, Bendigo, Victoria, Australia ${ }^{3}$ Department of Dentistry and Oral Health, La Trobe Rural Health School, La Trobe University, Bendigo, Victoria, Australia

${ }^{4}$ Lincoln International Institute for Rural Health, University of Lincoln, Lincoln, UK

Correspondence to Professor Amanda Kenny; a.kenny@latrobe.edu.au

\section{ABSTRACT}

Introduction Poor oral health among older people is a global problem impacting on health and well-being. The economic cost to the health system is significant. An ageing population is intensifying the urgency for action. However, poor oral health, particularly for those in residential aged care facilities, continues to be highly resistant to resolution. The overall aims of this realist review are to: $(A)$ explore and synthesise evidence on oral health interventions for older people in residential aged care facilities, (B) produce a causal theory on how contextual factors and mechanisms interact to produce outcomes, and (C) produce guidelines/policies to inform high-quality oral health interventions to improve older people's oral health in residential aged care facilities. Methods and analysis The review is guided by the RAMESES publication standards for realist synthesis. Participants include older people in residential aged care facilities, the aged care workforce, carers and families. Interventions include oral healthcare, oral health education, policy interventions and oral health promotion. The fivestep realist review process of Pawson et al will guide the review: clarification of scope and development of initial framework, systematic searches, study appraisal and data extraction, synthesising evidence, drawing conclusions, and dissemination, implementation and evaluation. Expert input with key stakeholders will occur through a blog. Stakeholders will examine consistencies across studies and an explanatory causal theory will be developed to guide policy and practice.

Ethics and dissemination Formal ethical approval was granted by the La Trobe University Ethics Committee HREC 20144. The developed theory will guide education, practice and policy decisions about interventions and the factors that impact on implementation. Using an integrated knowledge translation approach, traditional research outputs such as international conference presentations and publications will be supplemented with stakeholder forums, infographics, blogs, social media postings, webinars, podcasts and writing for webbased independent outlets.

PROSPERO registration number CRD42021155658.

\section{INTRODUCTION}

The overall aims of this realist review ${ }^{1-3}$ are to: (A) explore and synthesise evidence on oral health interventions for older people in
Strengths and limitations of this study

- No authors have undertaken a realist review to explore the impact of context and mechanisms on oral health outcomes for older people in residential aged care facilities.

- Realist reviews are an important strategy in evidence synthesis and serve to identify causal explanations of why interventions may work differently in different contexts, and how responses and reasoning influence outcomes.

- To maximise the usefulness of the review, integrated knowledge translation will occur throughout, with a range of perspectives captured from policymakers, commissioners, service providers, residents from aged care facilities, families and carers to ensure the relevance of the developed theory, guidelines and policies.

- Realist reviews involve an iterative approach to document context, mechanism and outcome, which can impact on reproducibility.

- Only studies published in English will be included in the review. We acknowledge this is a limitation that will impact on the findings as literature published in languages other than English will not be included.

residential aged care facilities, (B) produce a causal theory on how contextual factors and mechanisms interact to produce outcomes, and (C) produce guidelines/policies to inform high-quality oral health interventions to improve older people's oral health in residential aged care facilities.

Realism is built on the tenet that causal relationships and uniformities between interventions are established by exploring the various system inputs and outputs that impact on outcomes. Realist systematic reviews are an important strategy in evidence synthesis and serve to identify causal explanations of why interventions may work differently in different contexts, and how responses and reasoning influence outcomes. ${ }^{4}$ In realist systematic reviews, context refers to factors 
such as the culture and history of the intervention site, existing social networks, human resources, sources of funding and geographic location. Mechanisms are defined as the 'generative force that leads to outcomes' ${ }^{3}$ (p 134). A mechanism involves participants' cognitive and emotional responses or reactions and understanding is essential to move beyond what happened, to why it happened, for who and in what circumstances. ${ }^{3}$

The growing burden of oral disease among older people is a global public health problem. ${ }^{5}$ In 2007 , the WHO identified older people's oral health as an urgent priority for action. ${ }^{78}$ More than a decade later, it is a critical issue that is amplified by a rapidly ageing population. In 2015, Lewis and colleagues ${ }^{9}$ described oral health in residential aged care as a 'wicked problem'-difficult, complex and highly resistant to resolution because of intrinsic structural and mechanistic features ${ }^{10}$ With rising numbers of older people, the problem will intensify. A realist systematic review is appropriate to explore interventions to address older people's oral health because of the complexity of associated system issues. An understanding of how interventions might work with this group requires a detailed explanation of contextual factors and mechanisms of change and how causal relationships might result in different outcomes in different settings.

In 2017, there were 962 million older people globally, with predictions of 2.1 billion in 2050 and 3.1 billion in 2100. The number of people over 60 years is expected to more than double by 2050 and more than triple by 2100 . The most rapidly growing age group is those over 80 years. ${ }^{11}$ This group is expected to more than quadruple over the next four decades from less than 90 million in 2005 to almost 400 million by $2050 .{ }^{11}$ One estimate from Australia shows that the demand for aged care services is expected to increase by $250 \%$ over the next 40 years. ${ }^{9}{ }^{12}$ The WHO, international governments and policymakers continue to call for urgent public health action on older people's oral health to address a rapidly rising burden of disease. 81314

The main oral diseases and conditions impacting on older people are dental caries (or tooth decay), periodontal disease (a group of inflammatory diseases of the gum, connective tissue and dental bone), tooth loss, xerostomia (dry mouth) and oral precancer/cancer lesions. ${ }^{58}$ Over the last 50 years, significant effort has gone into understanding the relationship between oral health, general health and well-being. ${ }^{8}$ Compromised oral health is associated with respiratory and cardiovascular diseases, diabetes and stroke. ${ }^{15}$ Data on older people's oral healthrelated quality of life (OHRQoL) are limited, with little known about those over 80 years. ${ }^{16}{ }^{17}$ However, there is consensus that the impact of poor oral health extends beyond the systemic health of older adults and has a significant impact on people's overall QoL. ${ }^{18}$

Periodontal disease and dental caries are highly prevalent chronic, cumulative oral diseases in older people, with management complicated by varied levels and combinations of other chronic conditions and reduced functional ability. There is agreement that a tailored approach to oral healthcare is needed to meet the unique needs and dependency levels of older adults. ${ }^{18}$ Authors argue that further policy development targeting older people is required to address underlying environmental, economic and social causes of poor oral health in this group. ${ }^{18}$ Oral healthcare in older adults is impacted by circumstances of earlier life stages, including alcohol and tobacco use, access to oral health treatment and prevention, and food and beverage consumption. In older people, poor oral health can result in considerable pain, reduced chewing performance, and can impact on food choice, weight loss, impaired communication, low self-esteem and wellbeing. ${ }^{19-21}$ OHRQoL is poorer among those who wear dentures, have higher numbers of missing or decayed teeth or have dry mouth. ${ }^{22} 23$

Ageing is traditionally associated with edentulism (having no natural teeth), and in developed countries, oral healthcare in older people has mostly centred on denture care. However, this is changing with growing numbers of older people retaining some or most of their natural teeth and increasing numbers presenting with more complex tooth and implant-supported restorations and dentures. ${ }^{9}$ For example, in Australia, the percentage of people aged $75+$ wearing full dentures declined from $79 \%$ in 1979 to around $28 \%$ in 2010. ${ }^{94-26}$ Optimum oral healthcare for this group requires ongoing care and appropriate and timely intervention. In this review, oral health interventions that target both dentate and edentate older adults will be included.

Residential aged care settings present key challenges for oral healthcare. In this review, we define residential aged care as a facility that is specifically designed to provide accommodation for older people and supports for day-to-day living. The care provided in these facilities may include personal care or nursing care or both. Older people in residential aged care are often medically and cognitively compromised and polymedicated..$^{27} \mathrm{~A}$ combination of critical factors increases their risk of poor oral health. Commentators have observed that older people in residential aged care receive inadequate oral healthcare, and even where services do exist, oral health protocols and guidelines are mostly absent, and services are ad hoc or uncoordinated. There appears to be a lack of routine oral healthcare, inadequate assessments and poor documentation related to oral health. ${ }^{28}$ Personal care assistants (or equivalent) make up the bulk of staffing in residential aged care. Oral health has a low priority among residential care staff with lack of experience, attitudes such as fear and distaste, and an unwillingness to provide oral healthcare acting as significant barriers to quality care. ${ }^{29} 30$

While poor oral health among older people creates a massive burden at the individual level, ${ }^{2231} 32$ the economic cost is also significant. As an example, in Australia in 2013-2014, poor oral health was responsible for more than 8000 potentially preventable hospital admissions for people aged $65 .{ }^{33}$ In 2016-2017, recurrent 
expenditure on oral health services in Australia was $\$ 10.2$ billion, up from an inflation-adjusted $\$ 6.6$ billion in 2006-2007. ${ }^{34}$ Similar rates of potentially preventable hospital admissions have been reported internationally. ${ }^{35-38}$ However, the actual economic burden of poor oral health on all parts of the health system is not known. ${ }^{39}$

There have been several systematic reviews exploring oral health interventions for older people in residential care. ${ }^{40-45}$ These reviews have focused on specific oral health issues: prevalence of oral disease, ${ }^{44}$ assessment tools, ${ }^{44}$ effectiveness and impact of strategies to promote or improve oral health, ${ }^{42} 4345$ oral health education, ${ }^{44} 45$ barriers to oral healthcare ${ }^{46}$ cost of oral health, ${ }^{47}{ }^{48}$ role of oral healthcare in preventing pneumonia ${ }^{495}$ and the association with malnutrition. ${ }^{47}$ Specific populations include frail residents, ${ }^{43}$ those with dementia ${ }^{44} 51$ and caregivers such as nurses ${ }^{425253}$ and assistants. ${ }^{54}$

No authors have synthesised the evidence on oral health interventions for older people in residential aged care from a realist perspective. While the prevalence of oral disease among older people is well known, there are substantial differences in government policies, interventions, the availability of oral health interventions for older people and the knowledge and skill level of those delivering interventions. There are significant gaps in understanding how interventions work and whether causal relationships and uniformities can be used to produce theoretical understandings of how and why interventions work. This review directly addresses this gap and has the potential to transform oral health systems and ultimately build public health capacity. ${ }^{55}$

\section{REALIST REVIEW METHODOLOGY}

This realist systematic review focuses on oral health interventions for older people in residential aged care facilities. By grounding the review in critical realism, we can move beyond a more traditional approach to a systematic review $^{1-3}$ and capture the contextual factors and mechanisms of change that impact on the success of oral health interventions in residential aged care settings. Through consideration of context, mechanism and outcome we will explore successful and unsuccessful interventions and those with mixed results. ${ }^{1}$ A context-bound approach to causality is represented as context+mechanism=outcome. ${ }^{23}$ We hypothesise that the contexts in which oral health interventions are delivered in the residential aged care setting are complex, multifaceted and dynamic; interventions rarely work in the same way within different contexts.

Past reviews have focused mostly or exclusively on outcomes, with little attention to how the interplay between context and mechanism can impact an outcome. Understanding of this interplay is critical for future development and implementation of interventions and accelerating translation of evidence to practice. ${ }^{56}$

\section{METHODS AND ANALYSIS}

\section{Review aims and questions}

The overall aims of this realist review ${ }^{1-3}$ are to (A) explore and synthesise evidence on oral health interventions for older people in residential aged care, (B) produce a causal theory ${ }^{57-59}$ on how contextual factors and mechanisms interact to produce outcomes, and (C) produce guidelines/policies to inform high-quality oral health interventions to improve older people's oral health in residential aged care facilities. The review is guided by the Realist and Meta Narrative Evidence Synthesis: Evolving Standards (RAMESES) publication standards for realist synthesis. $^{2}$

The review questions are:

1. What contextual factors impact on the outcomes of oral health interventions for older people residing in residential aged care?

2. What are the underlying mechanisms that contribute to intervention outcomes?

3. How do the mechanisms and contextual factors interact to produce intended and unintended outcomes from the interventions?

4. Is there consistency of contextual factors and mechanisms of change across studies?

5. How can the examination of context, mechanism and outcomes of oral health interventions in residential aged care be used to develop an explanatory causal theory to guide policy and practice?

The review protocol is registered with PROSPERO. The anticipated start date of the review is 1 March 2021 and the anticipated completion date is 1 March 2023.

We will follow the five-step realist review process of Pawson et $a l^{l}$ : clarifying scope, searching for evidence, appraising primary studies and extracting data, synthesising evidence and drawing conclusions, and disseminating, implementing and evaluating.

\section{Stage 1: clarifying scope and developing an initial framework}

Consistent with other realist reviews, ${ }^{57} 60-62$ the review questions were refined through extensive interaction with the review team and were informed by our community interactions with older people. The team is multidisciplinary (consisting of oral health, nursing, public health, psychology, sociology, aged care and paramedicine) so a multitude of perspectives have been captured and included. The defining feature of realist reviews is theory development and refinement. We will adopt the approach of Pawson and colleagues to 'scavenge ideas', (S1:25) from an initial scope of the literature, including the grey literature, to develop a long list of contexts, mechanisms and outcomes evident in published interventions. We will group ideas into potentially relevant beginning theories and will cluster these to form an initial evaluative framework that will be populated in later stages.

In view of COVID-19 and Australian restrictions on faceto-face contact we will engage with a range of people with expertise and interest in older people and oral health (eg, policymakers, commissioners, service providers, residents 
from aged care facilities, families and carers) via webbased blog technology. A blog site has been developed (www.olderpeopleoralhealth.wordpress.com) using the open-source blogging platform, WordPress.com. Ethical approval for the use of this site has been granted by the University Ethics Committee (HREC 20144). Blogs have been used successfully in a range of research projects ${ }^{63-66}$ and can support integrated knowledge translation (iKT) ${ }^{67}$ We are experienced at facilitating stakeholder engagement via a blog and have published on the usefulness of this approach for research data collection. ${ }^{6566}$

The purpose of the blog will be to tap into 'official conjecture $^{1}(\mathrm{~S} 1: 26)$ on why oral health interventions might work in different residential aged care settings and how context (culture and history, social networks, human resources, funding and settings) and mechanisms (participants' cognitive and emotional responses or reactions, motivations, understandings, beliefs and attitudes) might impact on outcomes. We will widely advertise the blog through existing national and international professional and consumer networks, print and social media. The blog will be open for the duration of the project and people will be invited to participate at a time that suits them. Participants will have the choice to post with their name visible or they can choose to be anonymous. Stakeholder views gathered from the blog will be fed into our initial evaluative framework and we will seek input into all stages of the review to test and refine theories as they emerge. The approach aligns strongly with $\mathrm{iKT}^{68}{ }^{69}$ where researchers and those who will be end users of research work together to produce findings.

\section{Stage 2: searching for evidence}

In the searching for evidence stage, we will work with a specialist healthcare librarian to complete systematic searches. The following key concepts outlined in table 1 have been developed to guide our search.

Searches will be completed in each database (MEDLINE Ovid, Embase Ovid, PsycINFO, Academic Search Complete, CINAHL EBSCO, Cochrane Oral Health Trials Register, Cochrane Central Register of Controlled Trials (CENTRAL)) based on the one developed for MEDLINE (see table 2).
Medical Subject Headings terms will guide the search, and search terms will include truncation of keywords, use of thesaurus terms and subject headings. Terms will be developed into search strings with the appropriate Boolean operators.

A four-phase search process will be undertaken: (A) title and abstract, (B) full text, (C) search of the reference list of included studies and consideration of any further studies as at phases A and B, and then (D) search of citations for all included studies and consideration of any further studies as at A and B. These phases will ensure breadth of capture.

A broad approach to capturing grey literature will be undertaken. Google, Google Scholar and the Bielefeld Academic Search Engine will be used to source reports, working and white papers, government documents and evaluations of interventions. Targeted searching will occur on relevant websites, including dental professional bodies and government agencies. A search of library databases that index relevant grey literature will be undertaken. This will include searches of Embase, the Cochrane Library, and ProQuest Dissertations and Theses. Published conference papers will be searched using COS: Conference Papers Index (via ProQuest) and Scopus. Forward searching of citations of relevant journal articles will be completed to identify current works. Social media and the blog will be used to locate other relevant sources.

Pawson et al identify a key difference between realist reviews and conventional systematic reviews. They highlight the iterative and interactive nature of searching in realist reviews and the need for breadth in study inclusion as the 'mechanism of action' is central in analysis. They do, however, highlight the importance of the deliberative use of purposive sampling to manage the potential for a very high number of studies to be identified and to know when to stop searching. Consistent with their guidelines, in this review, a purposive sampling approach will be undertaken to identify the best sources of evidence to understand how context and mechanisms influence the outcomes produced from oral health interventions for older people in residential care and to support theory development on causation, agency, structure and

\section{Table 1 Key concept development}

\begin{tabular}{llll}
\hline Concept $\mathbf{1}$ & Concept $\mathbf{2}$ & Concept $\mathbf{3}$ & Concept 4 \\
\hline Oral health & Residential care & Older people & Programme development \\
Oral hygiene & Nursing home & Aged & Oral health promotion \\
Dental health & Long-term care & Senior & Programme evaluation \\
Dental caries & Social care & Geriatric & National health programme \\
Dental health & Aged care home & Health policy \\
Dental hygiene & Home for the aged & Oral health education \\
Oral care & Residential facilities & Oral health programme \\
Dental care & Homes for the aged & Dental health services \\
\hline
\end{tabular}


Table 2 Example search string for MEDLINE

\begin{tabular}{|c|c|}
\hline Search No & Concepts \\
\hline S1 & AB 'Oral health’ OR 'Oral hygiene’ OR ‘Dental caries' OR ‘Dental care' OR ‘Dental health' \\
\hline $\mathrm{S} 2$ & $\begin{array}{l}\text { AB (‘Oral health’ OR ‘Oral hygiene’ OR ‘Dental caries’ OR ‘Dental care’ OR ‘Dental health’) OR TI (‘Oral health' OR } \\
\text { 'Oral hygiene' OR ‘Dental caries' OR ‘Dental care’ OR ‘Dental health’) }\end{array}$ \\
\hline S3 & MH ('Oral Health’) \\
\hline S4 & dental health or oral health or dental hygiene or oral hygiene \\
\hline S5 & $\begin{array}{l}\text { AB ('resident" care' OR 'nursing home*' OR 'long term care*' OR 'social care' OR 'aged care home*' OR 'home for } \\
\text { the aged') OR TI ('resident* care' OR 'nursing home*” OR 'long term care*' OR 'social care' OR 'aged care home*' } \\
\text { OR 'home for the aged') }\end{array}$ \\
\hline S8 & (MH ‘Residential Facilities+’) \\
\hline S12 & AB (aged or senior* or 'older people’ or geriatric*) OR TI (aged or senior* or 'older people' or geriatric*) \\
\hline S13 & (MH 'Aged+') \\
\hline S14 & (((MH ‘Dental Care for Aged’)) AND (S12 OR S13)) AND (S12 OR S13) \\
\hline S15 & 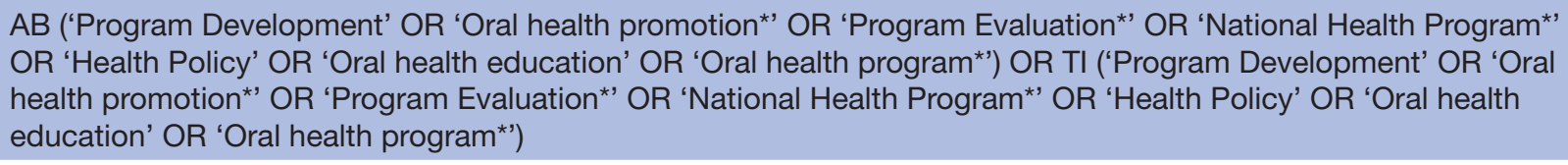 \\
\hline S16 & (MM ‘Dental Health Services') OR (MM ‘Health Education, Dental’) OR (MH ‘Health Promotion+') \\
\hline $\mathrm{S} 17$ & (MH 'Dental Care for Aged’) \\
\hline S18 & ((MH ‘Dental Care for Aged’)) AND (S15 OR S16 OR S17) \\
\hline
\end{tabular}

relationships. Through team discussion we will follow the guiding principle described by Burton et a $\ell^{62}$ to assess whether the evidence is 'good enough' to include at the synthesis stage. ${ }^{1}$ Team consensus and expert input via the blog will inform when saturation has been reached; 'when sufficient evidence has been assembled to satisfy the theoretical need or answer the question"70 (p 20). Two questions will be asked: (A) does the sourced literature add to understandings of the 'mechanism of action' of the intervention? and (B) is further searching likely to add to these understandings?

Search results will be imported to EndNote bibliographic software and then to Covidence, Cochrane's systematic review management software.

\section{Study designs}

Consistent with realist reviews, ${ }^{1-3}$ quantitative, qualitative and mixed methods studies will be included. There are no limitations on the data range. We will only include studies published in English. The English language limitation reflects the significant resource implications associated with translation; however, we do note this will be a limitation of this review. ${ }^{71}$

\section{Population and setting}

Our population of interest includes older people living in residential care facilities, the residential aged care workforce (including but not limited to nurses, doctors, allied healthcare staff, dentists, oral health therapists, hygienists, personal care workers and other non-professional staff) and older people's carers and families. Research undertaken in residential aged care in any geographical setting will be included.

\section{Interventions}

The review will include any interventions, including oral healthcare assessment or intervention, oral health education, or other oral health promotion activities (including policy-level interventions) that have been delivered in residential aged care settings. Oral health education programmes directed at staff, residents or their families and oral health hygiene promotion or skills training, or a combination of both will be included. Content of the interventions could include some or all of the following: oral health, oral disease and impact on health, diet interventions related to improving oral health, oral hygiene measures, best oral health practices for older people with 


\begin{tabular}{|c|c|c|}
\hline Criterion & Inclusion & Exclusion \\
\hline Population & People aged 65 years and over. & People younger than 65 . \\
\hline Interventions & $\begin{array}{l}\text { Oral health intervention within residential aged care } \\
\text { settings that measure oral healthcare assessment or } \\
\text { intervention, oral health education or other oral health } \\
\text { promotion activities (including policy-level interventions) } \\
\text { for older people. Oral health education programmes } \\
\text { directed at staff, residents or their families and oral } \\
\text { hygiene promotion or skills training, or a combination of } \\
\text { both will be considered. }\end{array}$ & $\begin{array}{l}\text { Interventions based in the hospital or community } \\
\text { setting. }\end{array}$ \\
\hline Language & Manuscripts written in English. & Non-English. \\
\hline Dates & All date ranges. & Not applicable. \\
\hline
\end{tabular}

natural dentition and dentures, oral hygiene promotion and skills training (both for staff and residents) adapted from ref 40. Inclusion and exclusion criteria are outlined in table 3 .

All included records will be managed in Covidence. All reviewers will initially assess a sample of 25 articles to ensure reliability in application of the inclusion and exclusion criteria. Any discrepancies will be resolved via discussion. All studies will be screened by a minimum of two reviewers. As screening is conducted, conflicts will be automatically identified by the Covidence software and these will be discussed by the review team until consensus is reached. The systematic searching will be guided by the Preferred Reporting Items for Systematic Review and Meta-Analysis Protocols (PRISMA-P) ${ }^{72}$ checklist and a PRISMA flow chart will document all stages of study selection.

\section{Stage 3: appraising primary studies and extracting data}

Realist reviews extend quality appraisal beyond hierarchies of evidence of methodological quality. ${ }^{257}$ No studies will be excluded based on methodological quality, as important nuanced information about the context, mechanism and outcome could be lost. Consistent with the guidelines of Wong et al, ${ }^{2}$ study appraisal will focus on two main criteria: (A) whether the study is relevant to the theory being explored and (B) whether the study is sufficiently rigorous to be credibly used in developing a causal theory. In-depth team discussion will support decisionmaking on study quality.

To ensure richness of evidence, conventional data extraction tables will be used in the first stage to document authors, population, study design and interventions. Data will be extracted on oral health status (tooth loss, dental caries, periodontal disease, oral hygiene status, xerostomia, mucosal lesions and pain) of residents measured by any tools used in the included studies (eg, Brief Oral Health Status Examination or Oral Health Assessment Tool that covers different oral hygiene categories ${ }^{73}$ ), oral health-related knowledge of staff or residents (or both) measured by any instruments that are used in the included studies, oral health-related attitude and behaviour of staff or residents (or both) measured by any instruments used in the included studies and OHRQoL of residents measured using any QoL tool 58 in the included studies (eg, any version of Oral Health Impact Profile, ${ }^{75}{ }^{76}$ Geriatric/General Oral Health Assessment Index ${ }^{77}$ or Dental Impact Profile ${ }^{78}$ ).

As the focus is on realist concepts of what works best under what circumstances, data will be extracted from each study on the contextual factors and mechanisms of change, how they were triggered and the impacts on outcomes.

Initially, the focus will be on sifting and sorting ${ }^{1}$ of relevant literature and will include the extraction of free text descriptions of key features of interventions and their context. Consistent with other realist reviews, ${ }^{6061}$ the next stage will involve extensive note taking and annotations to capture detailed information about how an intervention is supposed to work, and ideas on the interplay of context, mechanism and outcome when interventions are implemented. ${ }^{3}$

A combination of inductive and deductive analytical logic will be used in a non-linear way. ${ }^{1}$ All articles will be input into NVivo data management software and will be independently reviewed by a minimum of two reviewers. Relevant sections will be highlighted and coded and 
brought together into context, mechanism and outcome categories.

This process is informed by Jagosh $e t a l^{3}$ and will require detailed discussion between team members to gain consensus on how data align with categories. Overlap between categories is expected and both inductive and deductive reasoning will be used to further populate our framework.

\section{Stage 4: synthesising evidence and drawing conclusions}

Constant immersion with the articles and the evaluative framework will enable identification of the best causal explanation for an outcome based on recurring patterns across the context, mechanism and outcome categories. ${ }^{62}$ As the evaluative framework is refined, feedback will be sought via the website and blog.

Using abductive reasoning, we will draw on our refined evaluative feedback to synthesise the evidence to find the clearest and most likely explanations for how content and mechanisms influence the outcomes produced from oral health interventions for older people in residential care.

The final stage will involve the development of a middle-range theory to support understandings of the main components of critical realism: the nature of causation, agency, structure and relationships. Explanation and causal analysis is integral for engaging complex social problems; in this case, older people's oral health, and suggesting solutions for social change. ${ }^{79}$ Recommendations, guidelines and policies will be tested via the blog and website and through an end-of-project forum that will be advertised through the website, print and social media.

\section{Stage 5: dissemination, implementation and evaluation}

To maximise the usefulness of the review, $\mathrm{iKT}^{6880}$ will occur throughout. By engaging with broad stakeholders via a blog, recommendations will be developed that have been informed by practical understandings of the settings in which interventions have been implemented. The blog will be used to drive local, national and international contributions at all stages of the review, including theory development and development of recommendations. Consistent with the international focus on impact, ${ }^{81-83}$ the aim is to increase the relevance, usefulness, applicability and impact of the review beyond academic publications. As the review is completed, traditional research outputs, such as international conference presentations and publications, will be supplemented with stakeholder forums, infographics, blogs, social media postings, webinars, podcasts and writing for web-based independent outlets. The insights gained will form the focus of future grant applications to apply and evaluate recommendations in practice. By engaging with broad stakeholders, recommendations will be developed that have been informed by practical understandings of the settings in which interventions have been implemented.

\section{Patient and public involvement}

The Violet Vines Marshman Centre for Rural Health Research is underpinned by extensive public involvement. The focus of this protocol has been informed by our work with older people who have helped shape the review question. No members of the public were involved in the writing of this protocol, but public involvement is included in all stages of the review.

\section{Ethics}

Formal ethical approval was granted by the La Trobe University Ethics Committee HREC 20144.

Twitter Virginia Dickson-Swift @VDSwift and Emma Zadow @ekzadow

Contributors AK, VDS and MG conceptualised the study protocol and all authors (AK, VDS, CKYC, MM, MG, BC, BH, SF, LCH, SC, EZ, RJK) contributed to the development, writing and reviewing of the protocol document. All authors read and approved the final manuscript.

Funding The authors have not declared a specific grant for this research from any funding agency in the public, commercial or not-for-profit sectors.

Competing interests None declared.

Patient and public involvement Patients and/or the public were involved in the design, or conduct, or reporting, or dissemination plans of this research. Refer to the Methods section for further details.

Patient consent for publication Not required.

Provenance and peer review Not commissioned; externally peer reviewed.

Open access This is an open access article distributed in accordance with the Creative Commons Attribution Non Commercial (CC BY-NC 4.0) license, which permits others to distribute, remix, adapt, build upon this work non-commercially, and license their derivative works on different terms, provided the original work is properly cited, appropriate credit is given, any changes made indicated, and the use is non-commercial. See: http://creativecommons.org/licenses/by-nc/4.0/.

ORCID iDs

Virginia Dickson-Swift http://orcid.org/0000-0002-7728-2101

Lisa C Hanson http://orcid.org/0000-0003-1612-3315

\section{REFERENCES}

1 Pawson R, Greenhalgh T, Harvey G, et al. Realist review - a new method of systematic review designed for complex policy interventions. J Health Serv Res Policy 2005;10:21-34.

2 Wong G, Greenhalgh T, Westhorp G, et al. RAMESES publication standards: realist syntheses. BMC Med 2013;11:21.

3 Jagosh J, Pluye P, Wong G, et al. Critical reflections on realist review: insights from customizing the methodology to the needs of participatory research assessment. Research Synthesis Methods 2014;5:131-41.

4 Rycroft-Malone J, McCormack B, Hutchinson AM, et al. Realist synthesis: illustrating the method for implementation research. Implementation Science 2012;7:33.

5 Petersen PE, Bourgeois D, Ogawa $\mathrm{H}$. The global burden of oral diseases and risks to oral health. Bull World Health Organ 2005;83:661-9.

6 Petersen PE, Yamamoto T. Improving the oral health of older people: the approach of the who global oral health programme. Community Dent Oral Epidemiol 2005;33:81-92.

7 World Health Organization (WHO). Oral health: action plan for health promotion and integrated disease prevention. Geneva: WHO, 2007.

8 Petersen P, Kandelman D, Arpin S. Global oral health of older people - call for public health action. Community Dental Health 2010;27:257-68.

9 Lewis A, Wallace J, Deutsch A, et al. Improving the oral health of frail and functionally dependent elderly. Aust Dent J 2015;60:95-105.

10 Australian Public Service Commission. Tackling wicked problems: a public policy perspective, 2018. Available: https://www.apsc.gov.au/ tackling-wicked-problems-public-policy-perspective

11 United Nations. World population prospects: 2017 revision. New York: United Nations, 2017. 
12 Deloitte Access Economics. The future of aged care in Australia: national seniors Australia, 2010. Available: https://nationalseniors. com.au/research/health-and-aged-care/the-future-of-aged-care-inaustralia [Accessed 20 April 2021].

13 Petersen PE. Global policy for improvement of oral health in the 21 st century - implications to oral health research of World Health Assembly 2007, World Health Organization. Community Dent Oral Epidemiol 2009;37:1-8.

14 Petersen PE. World Health Organization global policy for improvement of oral health - World Health Assembly 2007. Int Dent J 2008;58:115-21.

15 Azarpazhooh A, Tenenbaum HC. Separating fact from fiction: use of high-level evidence from research syntheses to identify diseases and disorders associated with periodontal disease. J Can Dent Assoc 2012;78:103-5

16 Hoeksema AR, Peters LL, Raghoebar GM, et al. Oral health status and need for oral care of care-dependent indwelling elderly: from admission to death. Clin Oral Investig 2017;21:2189-96.

17 Kossioni AE, Hajto-Bryk J, Janssens B, et al. Practical guidelines for physicians in promoting oral health in frail older adults. J Am Med Dir Assoc 2018;19:1039-46.

18 Hyde S, Dupuis V, Mariri BP, et al. Prevention of tooth loss and dental pain for reducing the global burden of oral diseases. Int Dent $J$ 2017;67:19-25.

19 Kandelman D, Petersen PE, Ueda H. Oral health, general health, and quality of life in older people. Special Care in Dentistry 2008;28:224-36

20 Bennadi D, Reddy CVK. Oral health related quality of life. J Int Soc Prev Community Dent 2013;3:1-6.

21 Wright FAC, Law GG, Milledge KL, et al. Chewing function, general health and the dentition of older Australian men: the Concord health and ageing in men project. Community Dent Oral Epidemiol 2019;47:134-41.

22 Murray Thomson W. Epidemiology of oral health conditions in older people. Gerodontology 2014;31:9-16.

23 Masood M, Newton T, Bakri NN, et al. The relationship between oral health and oral health related quality of life among elderly people in United Kingdom. J Dent 2017;56:78-83.

24 Australian Institute of health and welfare (AlHW) dental statistics and research unit. The National survey of adult oral health 2004-06: cat. No den 175 dental statistics and research series no 39. Canberra: Canberra Australian Institute of health and welfare, 2008.

25 Australian Institute of Health and Welfare (AlHW). Oral health and dental care in Australia: key facts and figures cat. No. den 214. Canberra: Australian Institute of Health and Welfare (AlHW), 2011.

26 van der Putten G-J, Mulder J, de Baat C, et al. Effectiveness of supervised implementation of an oral health care guideline in care homes; a single-blinded cluster randomized controlled trial. Clin Oral Investig 2013;17:1143-53.

27 Coker E, Ploeg J, Kaasalainen S, et al. A concept analysis of oral hygiene care in dependent older adults. $J$ Adv Nurs 2013;69:2360-71.

28 Slack-Smith L, Durey A, Scrine S. Successful aging and oral health incorporating dental professionals into aged care facilities. Perth, WA: Centre of Research Excellence in Primary Oral Health Care, University of Western Australia, 2016.

29 Australian Institute of Health and Welfare (AlHW). Caring for oral health in Australian Residental care Canberra. Canberra: Australian Institute of health and welfare (AlHW), 2009.

30 Paley GA, Slack-Smith L, O'Grady M. Oral health care issues in aged care facilities in Western Australia: resident and family caregiver views. Gerodontology 2009;26:97-104.

31 Thomson WM, Smith MB, Ferguson CA, et al. Oral status, cognitive function and dependency among New Zealand nursing home residents. Gerodontology 2018;35:185-91.

32 Sischo L, Broder HL. Oral health-related quality of life: what, why, how, and future implications. J Dent Res 2011;90:1264-70.

33 Australian Institute of Health and Welfare (AlHW). Oral health and dental care in Australia: key facts and figures 2015. Canberra: AlH, 2016.

34 Australian Institute of Health and Welfare (AlHW). Oral health and dental care in Australia. cat. den 231. Canberra: AlHW, 2019.

35 Australian Institute of health and welfare (AlHW). Admitted patient care 2016-17: Australian hospital statistics health services series No. 84. cat. No. HSE 201. Canberra: AlHW, 2018

36 Quiñonez C, leraci L, Guttmann A. Potentially preventable Hospital use for dental conditions: implications for expanding dental coverage for low income populations. $J$ Health Care Poor Underserved 2011;22:1048-58.
37 Lorden AL, Jiang L, Radcliff TA, et al. Potentially preventable hospitalizations and the burden of healthcare-associated infections. Health Serv Res Manag Epidemiol 2017;4:233339281772110.

38 Whyman RA, Mahoney EK, Morrison D, et al. Potentially preventable admissions to new Zealand public hospitals for dental care: a 20year review. Community Dent Oral Epidemiol 2014;42:234-44.

39 Richardson B, Richardson J. End the decay: the cost of poor dental health and what should be done about it. Melbourne: Brotherhood of St Lawrence, 2011.

40 Albrecht M, Kupfer R, Reissmann DR, et al. Oral health educational interventions for nursing home staff and residents. Cochrane Database Syst Rev 2016:9:CD010535.

41 Wang T-F, Huang C-M, Chou C, et al. Effect of oral health education programs for caregivers on oral hygiene of the elderly: a systemic review and meta-analysis. Int J Nurs Stud 2015;52:1090-6.

42 Weening-Verbree L, Huisman-de Waal G, van Dusseldorp L, et al. Oral health care in older people in long term care facilities: a systematic review of implementation strategies. Int J Nurs Stud 2013;50:569-82

43 Coker E, Ploeg J, Kaasalainen S. The effect of programs to improve oral hygiene outcomes for older residents in long-term care: a systematic review. Res Gerontol Nurs 2014;7:87-100.

44 Chalmers JM, Pearson A. A systematic review of oral health assessment by nurses and carers for residents with dementia in residential care facilities. Spec Care Dentist 2005;25:227-33.

45 Hoben M, Kent A, Kobagi N, et al. Effective strategies to motivate nursing home residents in oral care and to prevent or reduce responsive behaviors to oral care: a systematic review. PLoS One 2017;12:e0178913.

46 Göstemeyer G, Baker SR, Schwendicke F. Barriers and facilitators for provision of oral health care in dependent older people: a systematic review. Clin Oral Investig 2019;23:979-93.

47 Van Lancker A, Verhaeghe S, Van Hecke A, et al. The association between malnutrition and oral health status in elderly in long-term care facilities: a systematic review. Int J Nurs Stud 2012;49:1568-81.

48 MacEntee MI, Kazanjian A, Kozak J-F, et al. A scoping review and research synthesis on financing and regulating oral care in long-term care facilities. Gerodontology 2012;29:e41-52.

49 Liu C, Cao Y, Lin J, et al. Oral care measures for preventing nursing home-acquired pneumonia. Cochrane Database Syst Rev 2018:9:CD012416.

50 Sjögren P, Wårdh I, Zimmerman $\mathrm{M}$, et al. Oral care and mortality in older adults with pneumonia in hospitals or nursing homes: systematic review and meta-analysis. J Am Geriatr Soc 2016;64:2109-15.

51 Yi Mohammadi JJ, Franks K, Hines S. Effectiveness of professional oral health care intervention on the oral health of residents with dementia in residential aged care facilities: a systematic review protocol. JBI Database System Rev Implement Rep 2015;13:110-22.

52 Chalmers J, Pearson A. Oral hygiene care for residents with dementia: a literature review. J Adv Nurs 2005;52:410-9.

53 Miegel K, Wachtel T. Improving the oral health of older people in long-term residential care: a review of the literature. Int J Older People Nurs 2009;4:97-113.

54 Hoben M, Clarke A, Huynh KT, et al. Barriers and facilitators in providing oral care to nursing home residents, from the perspective of care aides: a systematic review and meta-analysis. Int $J$ Nurs Stud 2017;73:34-51

55 Petersen PE, Ogawa $\mathrm{H}$. Promoting oral health and quality of life of older people - the need for public health action. Oral Health Prev Dent 2018:16:113-24.

56 Chan CKY, Oldenburg B, Viswanath K. Advancing the science of dissemination and implementation in behavioral medicine: evidence and progress. Int J Behav Med 2015;22:277-82.

57 Abayneh S, Lempp H, Manthorpe J, et al. Development of programme theory for integration of service user and caregiver involvement in mental health system strengthening: protocol for realist systematic review. Int J Ment Health Syst 2018;12:41.

58 O'Campo P, Molnar A, $\mathrm{Ng} \mathrm{E}$, et al. Social welfare matters: a realist review of when, how, and why unemployment insurance impacts poverty and health. Soc Sci Med 2015;132:88-94

59 Greenhalgh T, Kristjansson E, Robinson V. Realist review to understand the efficacy of school feeding programmes. BMJ 2007;335:858-61.

60 Lawal AK, Groot G, Goodridge D, et al. Development of a program theory for clinical pathways in hospitals: protocol for a realist review. Syst Rev 2019;8:136.

61 Keen J, Greenhalgh J, Randell R, et al. Networked information technologies and patient safety: a protocol for a realist synthesis. Syst Rev 2019;8:307. 
62 Burton C, Williams L, Bucknall T, et al. Understanding how and why de-implementation works in health and care: research protocol for a realist synthesis of evidence. Syst Rev 2019;8:194.

63 Lingetun L, Fungbrant M, Claesson I-M, et al. 'I just want to be normal' - A qualitative study of pregnant women's blogs who present themselves as overweight or obese. Midwifery 2017;49:65-71.

64 Murphy E, Donohue G, McCann E. Exploring mental health issues through the use of blogs: a scoping review of the research evidence. Issues Ment Health Nurs 2020;41:296-305.

65 Wilson E, Kenny A, Dickson-Swift V. Using blogs as a qualitative health research tool. Int J Qual Methods 2015;14:160940691561804.

66 Wilson E, Kenny A, Dickson-Swift V. Ethical challenges of community based participatory research: exploring researchers' experience. Int J Soc Res Methodol 2018;21:7-24.

67 Jagosh J, Macaulay AC, Pluye P, et al. Uncovering the benefits of participatory research: implications of a realist review for health research and practice. Milbank Q 2012;90:311-46.

68 Kothari A, Wathen CN. Integrated knowledge translation: digging deeper, moving forward. J Epidemiol Community Health 2017;71:619-23.

69 Kreindler SA. Advancing the evaluation of integrated knowledge translation. Health Res Policy Syst 2018;16:104.

70 Pawson P, Greenhalgh T, Harvey G, et al. Realist synthesis: an introduction. ESRC research methods programme, University of Manchester. RMP methods paper 2, 2004. https://www. betterevaluation.org/en/resources/guide/realist_synthesis_an_ introduction

71 Morrison A, Moulton K, Clark M. English-language restriction when conducting systematic review-based meta-analyses. Ottawa, Canada: Canadian Agency for Drugs and Technologies in Health, 2009
72 Moher D, Shamseer L, Clarke M, et al. Preferred reporting items for systematic review and meta-analysis protocols (PRISMA-P) 2015 statement. Syst Rev 2015;4:1.

73 Chalmers JM, King PL, Spencer AJ, et al. The oral health assessment tool--validity and reliability. Aust Dent J 2005;50:191-9.

74 Kayser-Jones J, Bird WF, Paul SM, et al. An instrument to assess the oral health status of nursing home residents. Gerontologist 1995;35:814-24.

75 Slade GD. Derivation and validation of a short-form oral health impact profile. Community Dent Oral Epidemiol 1997;25:284-90.

76 Slade GD, Spencer AJ. Development and evaluation of the oral health impact profile. Community Dent Health 1994;11:3-11.

77 Atchison KA, Dolan TA. Development of the geriatric oral health assessment index. J Dent Educ 1990;54:680-7.

78 Strauss RP. The dental impact profile. Measuring Oral Health and Quality of Life 1996;13:81.

79 Fletcher AJ. Applying critical realism in qualitative research: methodology meets method. Int J Soc Res Methodol 2017;20:181-94.

80 Gagliardi AR, Berta W, Kothari A, et al. Integrated knowledge translation (IKT) in health care: a scoping review. Implementation Science 2015;11:38.

81 Higgins JPT, Altman DG, Gøtzsche PC, et al. The Cochrane Collaboration's tool for assessing risk of bias in randomised trials. BMJ 2011;343:d5928.

82 Australian Research Council (ARC). Research impact: principles and framework Canberra Australian government, 2015. Available: https://www.arc.gov.au/policies-strategies/strategy/research-impactprinciples-framework

83 Greenhalgh T, Raftery J, Hanney S, et al. Research impact: a narrative review. BMC Med 2016;14:78. 